\title{
Feuchtigkeitsbestimmung durch parallele Messung von elektrischer Leitfähigkeit und Wasseraufnahme dünner SPEEK-Schichten
} Niklas Warnecke ${ }^{1}$, Hendrik Wulfmeier ${ }^{1}$, Holger Fritze ${ }^{1}$, Luca Pasquini', Philippe Knauth ${ }^{2}$

\author{
${ }^{1}$ Institut für Energieforschung und physikalische Technologien, TU Clausthal, Clausthal, Deutschland \\ ${ }^{2}$ Universität Aix-Marseille, CNRS, Madirel, Electrochemistry of Materials Group \\ Kontakt: niklas.warnecke@tu-clausthal.de
}

\section{Einleitung}

Zahlreiche moderne Anwendungen ebenso wie natürliche Vorgänge werden stark von Zusammensetzung der jeweilig vorhandenen Atmosphäre beeinflusst. In vielen Bereichen ist die Feuchtigkeit besagter Atmosphäre einer der präsentesten und bedeutendsten Faktoren. Folglich besteht ein hohes Interesse an einer präzisen Messung dieser, insbesondere mit leicht zu handhabenden Sensoren. Polymerelektrolyte sind hierbei vielversprechende Kandidaten, da sie eine hohe Robustheit gegenüber äußeren Einflüssen aufweisen und sie die Entwicklung kostengünstiger Sensoren bei gleichzeitig geringer Baugröße des Sensors ermöglichen.

Sulfoniertes Poly-Ether-Ether-Ketone (SPEEK) zeigt hier ein hohes Potential. PEEK ist ein mit Sulfonsäuregruppen versehenes Polymer mit der Summenformel $(\mathrm{C} 19 \mathrm{H} 12 \mathrm{O} 6 \mathrm{~S})_{\mathrm{DS}}(\mathrm{C} 19 \mathrm{H} 12 \mathrm{O})_{1-\mathrm{DS} .}$ Die Strukturformel ist in Abb. 1 dargestellt. DS steht hierbei für „Degree of Sulfonisation" bzw. Sulfonierungsgrad und gibt an, zu welchem Anteil das Polymer mit Sulfonsäuregruppen versehen wurde.

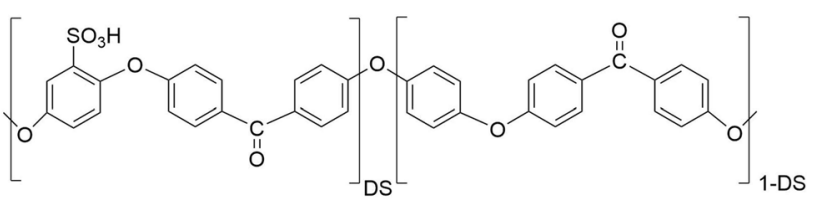

Abb. 1: Strukturformel von SPEEK

In feuchter Atmosphäre ist für SPEEK eine Protonenleitung über die Sulfonsäuregruppen möglich. Dort, wo sie am PEEK anliegen, kommt es zur Bildung hydrophiler Gebiete. Wenn SPEEK mit Feuchte in Kontakt kommt, nehmen diese Gebiete das Wasser auf und schwellen an [1]. Über diese Gebiete ist so Protonenleitung möglich.

Um SPEEK-basierte Feuchtigkeitssensoren zu realisieren, bieten sich folglich zwei Konzepte an, um die relative Atmosphärenfeuchtigkeit $r H$ zu bestimmen: (1) Messung der Massenzunahme/-abnahme $\Delta m$ mittels einer Quarzkristall-Nanowaage oder (2) Messung der Leitfähigkeit der Schichten $\sigma$ mittels Impedanzspektroskopie. Diese beiden Größen besitzen eine hohe Empfindlichkeit gegenüber Änderungen von $r \mathrm{H}$ auch über weite Bereiche von $\mathrm{rH}$ hinweg. Eine simultane Messung beider physikalischer Größen, $\Delta m$ und $\sigma$ erhöht die Sensitivität weiter, da sie die Anfälligkeit für Querempfindlichkeiten deutlich reduziert.
Da dünne SPEEK-Schichten eine schnelle Ansprechzeit auf $r \mathrm{H}$-Änderungen der Atmosphäre zeigen, wird so eine In-situ-Überwachung von Atmosphären oder auch eine direkte Prozessüberwachung ermöglicht.<smiles>Cc1ccc(Oc2ccc(Oc3ccc(C(=O)c4ccc(C)c(S(=O)(=O)O)c4)cc3)c(S(=O)(=O)O)c2)cc1</smiles>

Aufgenommenes Wasser<smiles>Cc1ccc(C(=O)c2ccc(Oc3ccc(C)cc3S(C)(=O)=O)cc2)cc1</smiles>

Abb. 2: Schema der Wasseraufnahme von SPEEK bei Sulfonsäuregruppen

Allerdings ist der zugrunde liegende Prozess der Wasseraufnahme und der zugehörige Zuwachs der Leitfähigkeit $\sigma$ von SPEEK bisher vor allem theoretisch betrachtet oder punktuell für einzelne $\mathrm{rH}$-Werte untersucht werden. Um eine praktischen (Sensor-)Anwendung zu ermöglichen, ist daher eine detaillierte Untersuchung unerlässlich. Insbesondere der Zusammenhang der beiden Messgrößen muss untersucht werden. Aus diesem Grund betrachtet die vorliegende Arbeit das Verhalten dünner SPEEKSchichten für wechselndes $r H$ im Detail. Dabei werden mit einer Nanowaage die Massenänderung $\Delta m$ und mittels Impedanzspektroskopie die Leitfähigkeit $\sigma$ bestimmt. Diese Messungen werden mit zwei SPEEK-Proben parallel durchgeführt. Der Prozess der Wasseraufnahme und die folgende Zunahme der Leitfähigkeit sowie der Zusammenhang dieser beiden können dadurch im Detail und in situ betrachtet werden.

Zudem wird aus den erhaltenen Werten der Zusammenhang zwischen der Konzentration der Protonen $c\left(H^{+}\right)$und ihrer Beweglichkeit $u\left(H^{+}\right)$ermittelt, um das Verständnis für den Prozess zu verbessern. 


\section{Materialien und Methoden}

\section{SPEEK}

Für die Arbeit werden zwei SPEEK Varianten mit unterschiedlichen Sulfonierungsgrad verglichen. Die DS-Werte betragen 0,5 und 0,9. Beide Varianten liegen in kristalliner Form vor und werden in Dimethylsulfonat (DMSO) nahe der Sättigungsgrenze gelöst.

\section{Nanowaage auf Basis von Langasit-Resonatoren $(\Delta m)$}

Um die Massenaufnahme zu messen, werden runde, piezoelektrische Langasit-Einkristalle (LGS, $\mathrm{La}_{3} \mathrm{Ga}_{5} \mathrm{SiO}_{14}$, Dichte zirka $5,754 \mathrm{~g} \mathrm{~cm}^{-3}$ ) mit einer Dicke von zirka $270 \mu \mathrm{m}$ und einem Durchmesser von $10 \mathrm{~mm}$ verwendet. LGS ist ein atmospähren- und hochtemperaturstabiler Quarzisomorph mit geringer elektrischer Leitfähigkeit. Auf diesen werden mithilfe der Laserablation beidseitig PlatinKontakte abgeschieden (Abb. 3 oben). Anschließend werden mit einer Einkanalpipette zirka 0,5 $\mu$ SPEEK-Lösung auf den Kontakt auf einer Seite aufgetragen. Diese Probe wird für zwei Stunden bei $80^{\circ} \mathrm{C}$ getrocknet. Auf dieselbe Weise SPEEK wird eine SPEEK-Schicht auf der anderen Seite der Probe aufgetragen und getrocknet. Ein Schema der hergestellten Probe ist in Abb. 3 dargestellt. Die beidseitige Beschichtung soll Verbiegungen des Resonators entgegenwirken, die durch auftretende Schichtspannungen bei der Aufnahme von Feuchtigkeit hervorgerufen werden können. Diese könnten das Messsignal verfälschen. Die Abmessungen der Schichten werden mithilfe eines taktilen Oberflächenprofilometers des Typs Ambios XP-2 (USA) vermessen.

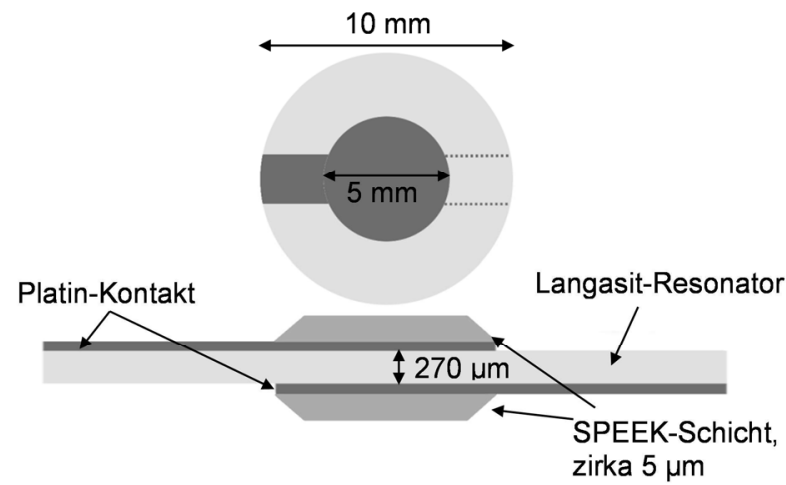

Abb. 3: Skizze eines Langasit-Resonators ohne SPEEK in Aufsicht (oben) und im Querschnitt mit SPEEK (unten)

\section{Aluminiumoxid-Einkristall $(\sigma)$}

Für die Leitfähigkeit werden Aluminiumoxid-Einkristalle (Saphir) mit einer Dicke von zirka $350 \mu \mathrm{m}$ verwendet. Auf diese werden per Siebdruck einseitig Interdigital-Strukturen aus Platin aufgebracht und anschließend für $1 \mathrm{~h}$ bei $1000^{\circ} \mathrm{C}$ ausgebacken. Analog zum Langasit-Kristall werden mithilfe einer Einkanalpipette zirka $0,5 \mu \mathrm{l}$ aufgetragen, sodass SPEEK zwischen die Kontakte gelangt (Abb. 4). Die Probe wird anschließend für zwei Stunden bei $80^{\circ} \mathrm{C}$ getrocknet. Die Abmessungen der Proben werden ebenfalls mittels des Profilometers ermittelt.

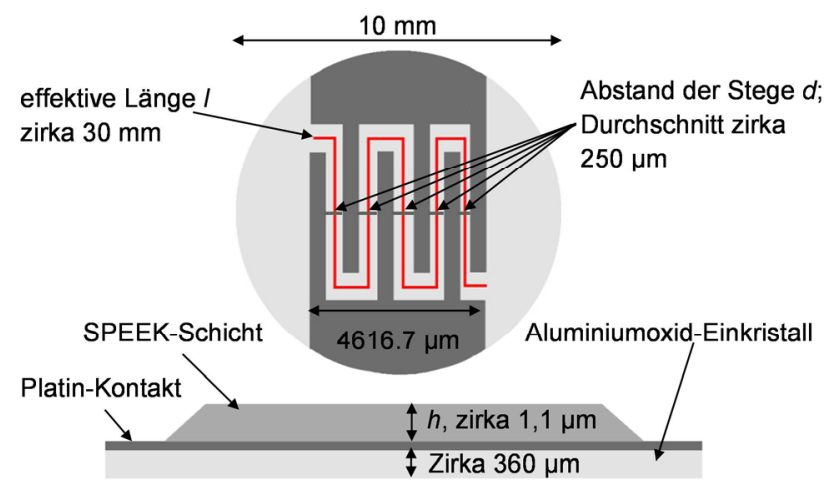

Abb. 4: Skizze eines präparierten AluminiumoxidEinkristalls ohne SPEEK in Aufsicht (oben) und im Querschnitt mit SPEEK (unten)

\section{Messaufbau}

Der gesamte Messaufbau ist in Abb. 5 skizziert. Für die Messung werden beide Varianten der Proben parallel in zwei Quartzglashalterungen eingelegt und über ihre Kontakte mit einem Netzwerkanalysator des Typs HP E5100A (USA) (Langasit-Kristall) und einem Impedanzanalysator vom Typ Solatron SI 1260 (USA) gekoppelt mit einem dielektrischen Interface Typ Solatron SI 1296 (Aluminiumoxid-Kristall) respektive verbunden. Ein Glaszylinder wird über die Apparatur gestülpt und gasdicht versiegelt, um so die Atmosphäre an den Proben von der Umgebung zu trennen. Ein Gasanschluss befindet sich am Deckel des Zylinders, um die Atmosphäre an den Proben zu verändern. Hierzu wird feuchte Luft durch diesen Anschluss in die Apparatur eingeleitet, indem Luft durch eine mit destilliertem Wasser gefüllte Gaswaschflasche gepumpt wird und somit mit Feuchtigkeit angereichert wird. Eine weitere Gaswaschflasche dahinter dient dazu, den Zufluss an Wasser in die Anlage zu verhindern. Beide Gaswaschflaschen befinden sich im Bad eines Umwälzthermostaten vom Typ Julabo F 33-ME (Deutschland). Indem die Badtemperatur verändert wird, kann die Wasseraufnahme der Luft reguliert und somit $\mathrm{rH}$ eingestellt werden. Die Atmosphärenfeuchtigkeit $\mathrm{rH}$ in der Messkammer wird mit einem am Boden des Messkorpus angebrachten Feuchtigkeitssensor C4.7 Galltec+mela (Deutschland) gemessen. Über denselben Anschluss kann die Luft auch mit trockenem Argongas (Reinheit $99,996 \%$ ) versetzt werden, um damit die Feuchtigkeit weiter zu reduzieren. Ein Massenflussregler regelt dabei die Zuflüsse. Am Boden der Anlage ist ein Einweg-Gasabfluss installiert.

Ein den Messkorpus einschließender Rohrofen Gero R050-250-13SO (Deutschland) hält zudem die Temperatur $T$ in der Messatmosphäre konstant auf zirka 
$30{ }^{\circ} \mathrm{C}$. Zur Kontrolle wird die Temperatur in der Messatmosphäre mithilfe eines Typ-S-Thermoelements und eines Pt100-Widerstandsthermometers gemessen.

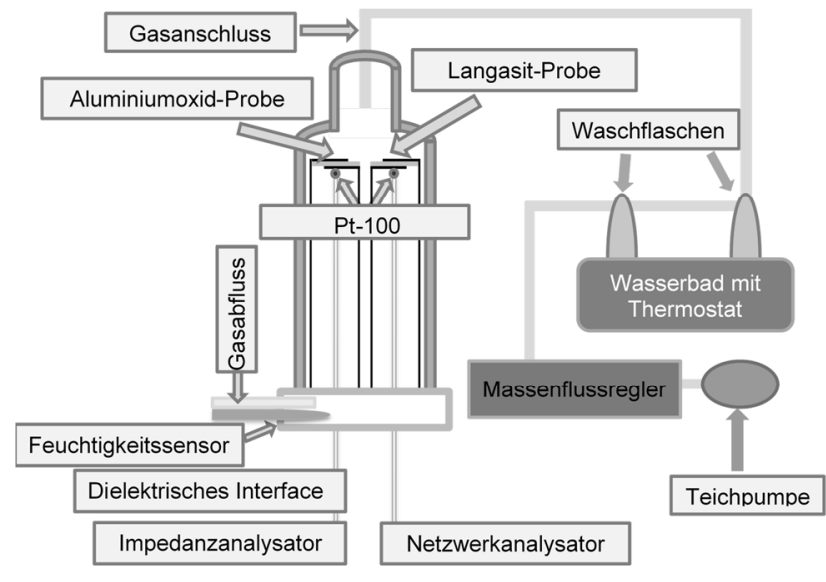

Abb. 5: Skizze der Messapparatur

\section{Messverlauf}

Für die Messung wird zunächst das Wasserbad erhitzt, bis der größtmögliche Wert für $r H$ erreicht wird (bei zirka $30^{\circ} \mathrm{C}$ ). Dieser Zustand wird für zirka 8 Stunden gehalten, um die Proben SPEEK-Schichten mit Feuchtigkeit zu sättigen und die Proben zu äquilibrieren. Anschließend wird dieser Prozess bei niedrigeren Temperaturen des Wasserbads und somit kleineren $\mathrm{rH}$-Werten wiederholt. Die einzelnen Messstufen werden stets für mindestens 6 Stunden gehalten. Bei $4{ }^{\circ} \mathrm{C}$ ist keine weitere Verminderung der Temperatur des Wasserbads möglich. Es werden kleinere $\mathrm{rH}$-Werte erreicht, indem zuerst die Messatmosphäre partiell durch Argon ersetzt wird: Der Durchfluss beträgt zuerst $20 \mathrm{ml} \mathrm{min}^{-1} \mathrm{Ar}$ und $50 \mathrm{ml} \mathrm{min}^{-1}$ Luft, anschließend $20 \mathrm{ml} \mathrm{min}^{-1} \mathrm{Ar}$ und $20 \mathrm{ml} \mathrm{min}^{-1}$ Luft und hiernach reines $\mathrm{Ar}$, jeweils für einige Stunden. Anschließend wird die Messatmosphäre mit einer Vakuumpumpe abgepumpt, sodass die SPEEK-Schichten zusätzlich getrocknet werden. Daraufhin wird wieder feuchte Luft eingelassen und schrittweise $r H$ über die Temperatur des Wasserbads erhöht.

\section{Nanowaage}

Zur Messung von $\Delta m_{H 2 O}$ des SPEEKs durch Wasseraufnahme wird der Langasit-Kristall als piezoelektrischer Resonator genutzt. Der Kristall wird zur Schwingung angeregt. Dabei ändert sich die Resonanzfrequenz anhand der Massenänderung. Für dünne Schichten kann hierzu die Sauerbrey-Gleichung (1) herangezogen werden [2]:

$$
\Delta m=-A_{E} \frac{\Delta f}{S_{m}}
$$

$\Delta m:$ Massenaufnahme

$A_{E}$ : effektive Fläche des SPEEK auf den Elektroden $\Delta f$ : Änderung der Resonanzfrequenz durch $\Delta m$ $S_{m}:$ Massensensibilität des Resonators
Die Massensensibilität $S_{M}$ ergibt sich aus dem Zusammenhang (2) [3]. Für diese Messung liegt sie im Bereich von $3320 \mathrm{~mm}^{2} \mathrm{\mu g}^{-1}$.

$$
S_{m}=\frac{f_{R}}{d * \rho}
$$

$f_{R}:$ Resonanzfrequenz des Resonators

$d$ : Dicke des Resonators

$\rho$ : Dichte von $L G S$

Die effektive Fläche $A_{E}$ ist von der realen Fläche $A_{R}$ zu unterscheiden [3]. Dies erfordert eine Korrektur nach (3) [4]:

$$
\frac{A_{E}}{A_{R}}=0,47-2,04 * 10^{-4} \frac{1}{{ }^{\circ} \mathrm{C}} * T
$$

Die Messung erfolgt bei $30{ }^{\circ} \mathrm{C}$, sodass sich ein Korrekturfaktor der realen Fläche zur effektiven Fläche von 0,464 ergibt, mit dem die reale Fläche zum Erhalt der effektiven Fläche zu multiplizieren ist.

Um zudem die Massenänderung durch die Aufnahme von Wasser relativ zur SPEEK-Masse bestimmen zu können, muss zunächst die trockene SPEEK-Masse bestimmt werden. Hierzu wird von den Proben die Resonanzfrequenz vor Auftragung von SPEEK gemessen und mit der Resonanzfrequenz in der mit Argon getrockneten Probe (siehe Messverlauf) verglichen. Mithilfe von (1), (2) und (3) lässt sich die Massenänderung in Folge der Aufnahme von SPEEK $\Delta m_{\text {SPEEK }}$ ermitteln. Die relative Massenänderung $\Delta m_{H 2 O}$,rel kann ermittelt werden, indem der Quotient aus der absoluten Massenänderung und diesem Wert gebildet wird (4).

$$
\Delta m_{H 2 O, r e l}=\frac{\Delta m_{H 2 O}}{\Delta m_{S P E E K}}
$$

$f_{R}$ : Resonanzfrequenz des Resonators

$d$ : Dicke des Resonators

$\rho$ : Dichte von $L G S$

\section{Nyquist-Diagramm}

Die Messung der Leitfähigkeit von SPEEK erfolgt, indem das Nyquist-Diagramm für die Probe aufgenommen wird. Dabei wird für die gemessenen Werte das Ersatzschaltbild aus Abb. 6 genutzt. Der Widerstand der SPEEK-Schicht ist dabei $R_{2}$. Die Daten werden bei verschiedenen $r H$ Werten gefittet, um $\mathrm{R}_{2}$ für diese zu ermitteln.

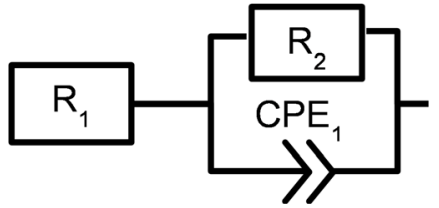

Abb. 6: Ersatzschaltbild für das Nyquist-Diagramm bestehend aus zwei Widerständen $\left(\mathrm{R}_{1}\right.$ und $\left.\mathrm{R}_{2}\right)$ und einem Konstant-Phasenelement $\left(\mathrm{CPE}_{1}\right)$ 
Die elektrische Leitung findet durch die SPEEK-Schicht zwischen den Stegen der Interdigitalstruktur mit dem Abstand $d$ statt. Somit erfolgt die Leitung durch die Querschnittsfläche der SPEEK-Schicht, die sich zwischen den Stegen befindet. Diese ergibt sich aus der Höhe der SPEEK-Schicht $h$ und der effektiven Länge der Strecke zwischen den Stegen I (siehe Abb. 4). So können in Verbindung mit der Probengeometrie aus den gemessenen Werten für $\mathrm{R}_{2}$ die zugehörigen Leitfähigkeiten der SPEEKSchicht ermittelt werden (5).

$$
\sigma=\frac{d}{A} * \frac{1}{R_{2}}=\frac{d}{l h R_{2}}
$$

$\sigma:$ Leitfähigkeit

A: Querschnittsfläche des SPEEKS

zwischen Kontakten

$h$ : Höhe der SPEEK - Schicht

l: effektive Länge der Querschnittsfläche

$d$ : Abstand zwischen den Kontakten

$R_{2}$ : Widerstand $R_{2}$ gemäß Abb. 6

\section{Konzentration und Beweglichkeit der Protonen}

Aus den erhaltenen Messdaten für $\Delta m_{H 2 O}$ lässt sich die Protonenkonzentration $c\left(\mathrm{H}^{+}\right)$für den zugehörigen $\mathrm{rH}$-Wert berechnen (6).

$$
c\left(H^{+}\right)=\frac{I E C(D S)}{100 * \Delta m_{H_{2} O, r e l}}
$$

IEC:Ion - Exchange - Capacity des SPEEK

$$
\begin{aligned}
& \operatorname{IEC}(D S=0,5)=1,5 \frac{\mathrm{meq}}{\mathrm{mg}} \\
& \operatorname{IEC}(D S=0,9)=2,5 \frac{\mathrm{meq}}{\mathrm{mg}}
\end{aligned}
$$

Aus der Protonenkonzentration $c\left(\mathrm{H}^{+}\right)$kann dann mithilfe von $\sigma$ die Beweglichkeit $u\left(H^{+}\right)$berechnet werden (7).

$$
u\left(H^{+}\right)=\frac{\sigma}{96,5 * c\left(H^{+}\right)}
$$

\section{Ergebnisse}

\section{Massenaufnahme}

Die auf den Langasit-Substraten aufgetragenen SPEEKSchichten zeigen anhand der Nanowaage eine trockene Masse $\Delta m_{\text {SPEEK }}$ von $86,76 \mu$ für DS 0,5 und $118,15 \mu \mathrm{g}$ für DS 0,9 .

Es werden $\mathrm{rH}-$ Werte im Bereich von zirka 3 bis $75 \%$ erreicht. Bei DS 0,9 konnten nur zirka $70 \%$ eingestellt werden. Über (4) kann mit diesen Werten $\Delta m_{H 2 O}$,rel ermittelt werden. Es ergeben sich die in Abb. 7 und Abb. 8 dargestellten Werte für $\Delta m_{H 2 O}$ und $\Delta m_{H 2 O}$,rel. Beide Werte nehmen näherungsweise linear mit der relativen Feuchtigkeit der Umgebung zu. Ebenso ist sichtbar, dass diese Aufnahme bei dem höheren DS von 0,9 in größerem Maße geschieht, als bei dem geringeren DS von 0,5. Es werden bei DS 0,5 bis zu zirka 11,9 $\mu$ g bzw. $14 \%$ bezüglich der trockenen SPEEK-Masse an Wasser aufgenommen. Dies wird bei $r H$ von $75 \%$ erreicht. Für DS 0,9 werden zirka $32,4 \mu \mathrm{g}$ bzw. $27,5 \%$ bei $r H$ von $70 \%$ erreicht.

Bei einer relativen Feuchtigkeit von zirka 51,5\% ist zudem ein Abfall in der Aufnahme von Wasser bei DS 0,5 zu geringeren Massen aufgenommenen Wassers festzustellen.

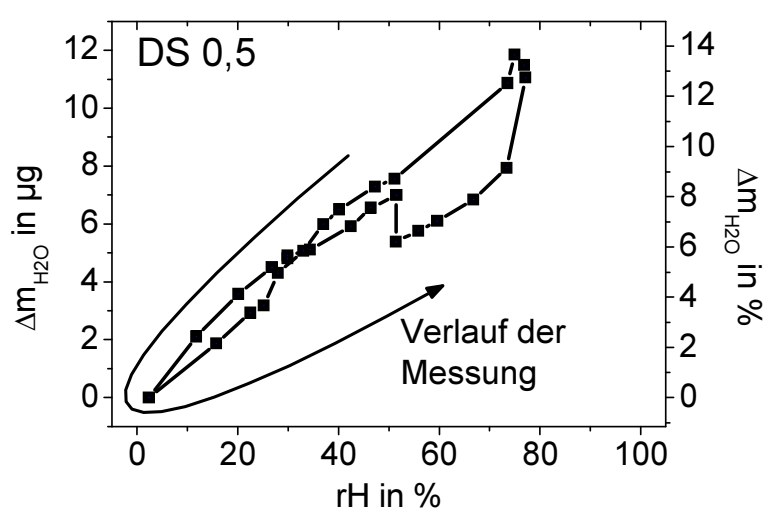

Abb. 7: Gemessene Massenaufnahme über relativer Feuchtigkeit für DS 0,5

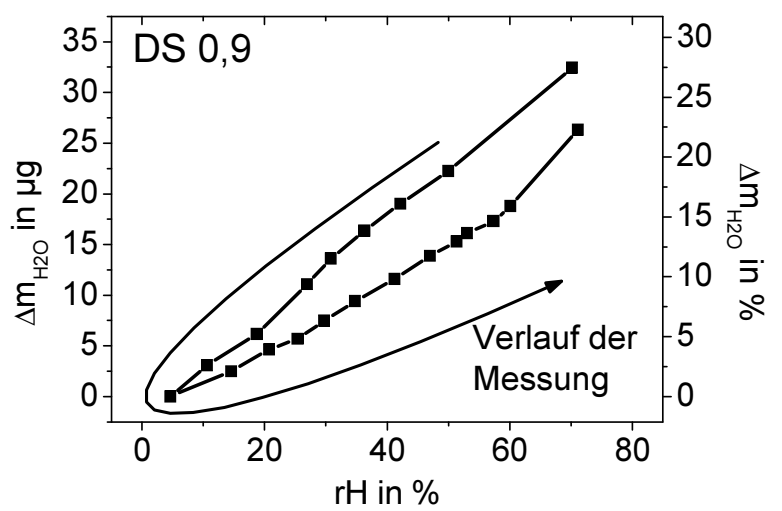

Abb. 8: Gemessene Massenaufnahme über relativer Feuchtigkeit für DS 0,9

\section{Leitfähigkeit}

Die Werte für die elektrische Leitfähigkeit sind in Abb. 9 und Abb. 10 für DS 0,5 und DS 0,9 respektive dargestellt. Es ist erkennbar, dass $\sigma$ exponentiell mit $r H$ ansteigt. Dabei liegen bei DS 0,9 die größeren Werte vor. Auch scheint $\sigma$ nach der Trocknung, wie zuvor $\Delta m_{H 2 O}$ und $\Delta m_{H 2 O}$,rel, langsamer anzusteigen. Es werden bei DS 0,5 Werte für $\sigma$ im Bereich von $7 \cdot 10^{-5} \mathrm{~S} \mathrm{~cm}^{-1}$ bei $\mathrm{rH}$ von zirka $75 \%$ erreicht. Bei DS 0,9 hingegen werden Werte für $\sigma$ von $4 \cdot 10^{-4} \mathrm{~S} \mathrm{~cm}^{-1}$ bei $\mathrm{rH}$ von zirka $70 \%$ erreicht. 


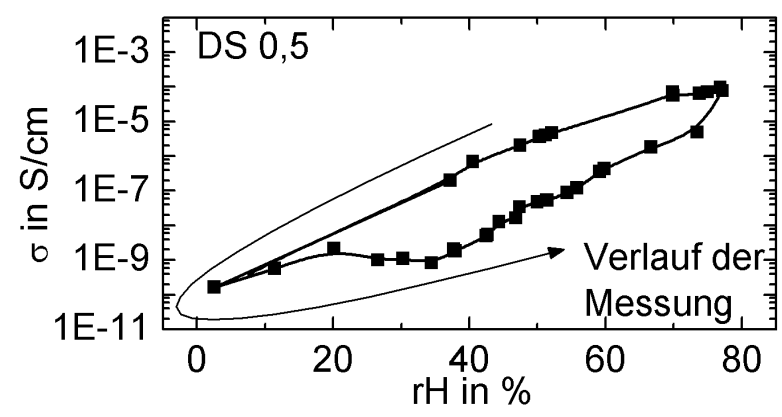

Abb. 9: Gemessene Leitfähigkeit über relativer Feuchtigkeit für DS 0,5

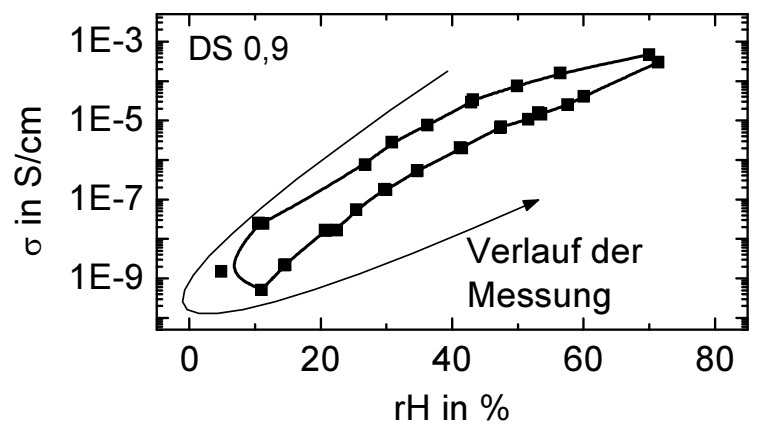

Abb. 10: Gemessene Leitfähigkeit über relative Feuchtigkeit für DS 0,9

Weiterhin werden die erhaltenen Werte für $\Delta m_{H 2 O}$,rel mit den erhaltenen Werten für $\sigma$ in Abb. 11 für beide DS-Werte korreliert. Es ergibt sich ein weitestgehend exponentieller Anstieg von $\sigma$ über $\Delta m_{H 2 O}$,rel.

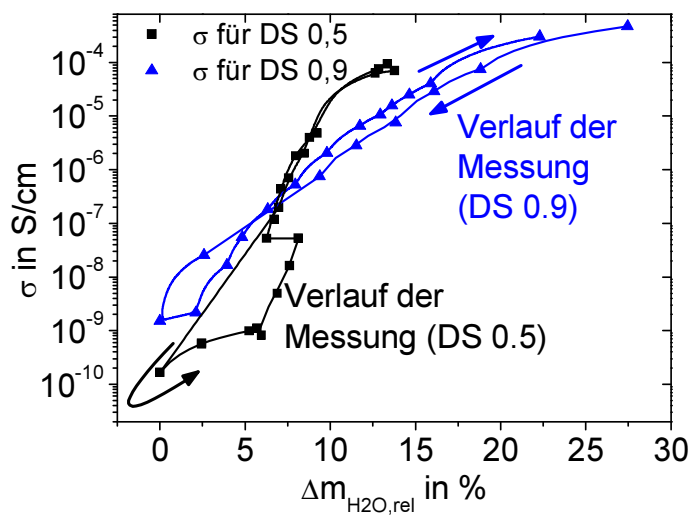

Abb. 10: Korrelation der gemessenen Leitfähigkeit mit der gemessenen Massenaufnahme

\section{Konzentration und Beweglichkeit der Protonen}

Über (6) und (7) können $c\left(H^{+}\right)$und $u\left(H^{+}\right)$aus den erhaltenen Werten berechnet werden. Die resultierende Änderung der Beweglichkeit der Protonen mit ihrer Konzentrati- on ist in Abb. 11 für beide DS-Werte ersichtlich. Dabei sind näherungsweise zwei Bereiche mit jeweils unterschiedlich starkem, exponentiellem Abfall der Beweglichkeit der Protonen mit höherer Konzentration feststellbar. Die Grenze zwischen diesen beiden Bereichen scheint bei $25 \mathrm{~g} \mathrm{~mol}^{-1} \mathrm{zu}$ liegen. Bei DS 0,5 werden dabei Werte für $u\left(H^{+}\right)$von bis zu zirka $8 \cdot 10^{-8} \mathrm{~cm}^{2} \mathrm{~s}^{-1} \mathrm{~V}^{-1}$ bei $c\left(H^{+}\right)$von zirka $11 \mathrm{~g} \mathrm{~mol}^{-1}$ erreicht. Bei DS 0,9 liegen die höchsten Werte für $u\left(H^{+}\right)$hingegen bei $5,4 \cdot 10^{-7} \mathrm{~cm}^{2} \mathrm{~s}^{-1} \mathrm{~V}^{-1}$ bei $c\left(H^{+}\right)$von zirka $9 \mathrm{~g} \mathrm{~mol}^{-1}$.

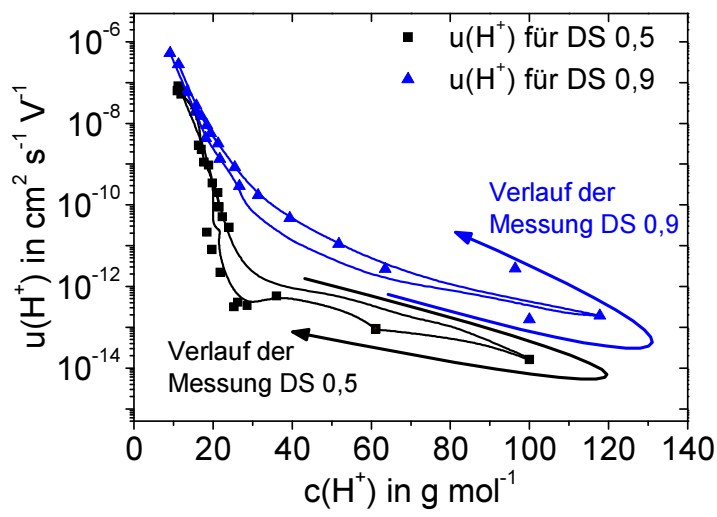

Abb. 11: Korrelation der Beweglichkeit der Protonen im SPEEK mit der zugehörigen Konzentration

\section{Diskussion}

Zwischen der Aufnahme von Wasser (relativ und absolut) und der relativen Feuchtigkeit der Umgebung besteht näherungsweise ein linearer Zusammenhang, während die Leitfähigkeit stattdessen exponentiell mit der relativen Feuchtigkeit zunimmt.

Die höhere Aufnahme an Wasser und folglich höhere Leitfähigkeit bei DS 0,9 statt DS 0,5 ist zu erwarten, da der Ladungstransport über die mit $\mathrm{H}_{2} \mathrm{O}$ gesättigten Sulfonsäuregruppen erfolgt. Da bei DS 0,9 mehr von diesen vorliegen, ist es naheliegend, dass diese SPEEK-Variation mehr Wasser aufnimmt und folglich besser leitet. Bei gleicher relativen Menge an aufgenommenen Wasser bilden sich aber bei beiden DS-Varianten ähnliche Werte für die Leitfähigkeit wie Abb. 10 zeigt.

Es scheint beim Trocknen ein Prozess stattzufinden, der die Fähigkeit zur Wasseraufnahme im SPEEK beschränkt. Dadurch steigt die aufgenommene Wassermenge weniger stark nach der Trocknung und folglich - da die Leitfähigkeit direkt von dem aufgenommenen Wasser abhängt steigt auch die Leitfähigkeit nach der Trocknung weniger stark an.

Der Abfall von DS 0,5 in Abb. 7 bei $51,5 \%$ relativer Feuchtigkeit scheint mit der geringeren Wasseraufnahme 
nach der Trocknung zusammenzuhängen: Der Verlauf vor dem Sprung liegt sehr nahe an dem Verlauf der Massenaufnahme vor dem Trocknungsvorgang, während der nachfolgende Verlauf niedriger ist, ähnlich wie bei DS 0,9. Es ist daher zu vermuten, dass der für die geringere Wasseraufnahme nach dem Trocknen verantwortliche Prozess bei DS 0,5 erst hier sichtbar wird, während er bei DS 0,9 sofort nach dem Trocknen erkennbar ist. Es ist unwahrscheinlich, dass dies auf eine höhere Wasseraufnahme bei DS 0,5 bis $51,5 \%$ zurückzuführen ist, die dann plötzlich abfällt. Denn es ist kein derartiger Sprung in Abb. 9 für die Leitfähigkeit erkennbar. Stattdessen liegen bei der Leitfähigkeit für beide DS-Werte geringere Werte nach der Trocknung vor. Eine genauere Untersuchung dieses Phänomens steht derzeit noch aus.

Quantitativ betrachtet sind die Werte für die relative Aufnahme von Wasser vergleichbar mit den Ergebnissen anderer Publikationen. Diese konzentrierten sich dabei auf $100 \%$ relative Feuchtigkeit oder sogar das Eintauchen von SPEEK in Wasser vor der Messung. Es wurden Massenaufnahmen von $23 \%$ bei DS 0,52 und $44 \%$ bei DS 0,81 [5] veröffentlicht. Ausgehend von einer linearen Steigung und keinerlei aufgenommenem Wasser bei $0 \%$ relativer Feuchtigkeit, würde dies für DS 0,5 bei $75 \%$ relativer Feuchtigkeit einer Aufnahme von 15,5\% entsprechen. Dies ist vergleichbar mit den Ergebnissen dieser Arbeit, da der Vergleichswert durch Eintauchen der Probe erreicht wurde, sodass der Wert für eine relative Feuchtigkeit von $100 \%$ nur näherungsweise angenommen werden kann. Bei DS 0,9 ergäbe dies 32,2\% Massenaufnahme bei $70 \%$ relativer Feuchtigkeit. Dies ist in Anbetracht der bereits genannten Gründe vergleichbar mit dem in dieser Arbeit ermittelten Wert von $27,5 \%$.

Auch die Leitfähigkeit entspricht den Ergebnisse von anderen Publikationen für eine relative Feuchtigkeit von $100 \%$ bzw. für SPEEK, das vor der Messung eingetaucht wurde: Die Werte liegen bei $10^{-3} \mathrm{~S} \mathrm{~cm}^{-1}$ für DS 0,52 und bei $10^{-2} \mathrm{~S} \mathrm{~cm}^{-1}$ für DS 0,81 [5]. Die hier gemessenen, maximalen Werte sind hingegen $7 \cdot 10^{-5} \mathrm{~S} \mathrm{~cm}^{-1}$ für DS 0,5 und $4 \cdot 10^{-4} \mathrm{~S} \mathrm{~cm}^{-1}$ für DS 0,9. Sie sind bei $75 \%$ (DS 0,5) und $70 \%$ (DS 0,9) relativer Feuchtigkeit gemessen und daher um zirka 1,5 Größenordnungen kleiner als die Vergleichswerte. Angesichts des in Abb. 9 und Abb. 10 sichtbaren Verlaufs, ist dieser Unterschied realistisch.

Die Änderung der Beweglichkeit als Funktionen der Konzentration in Abb. 10 bei zirka $25 \mathrm{~g} \mathrm{~mol}^{-1}$ ist möglicherweise auf den Sprung der aufgenommenen Wassermenge bei $51,5 \%$ relativer Feuchtigkeit zurückzuführen. Die hier gemessenen Messwerte für die Aufnahme an Wasser sind jene, die zur Berechnung der Konzentration bei zirka $51,5 \%$ herangezogen werden.

\section{Schlussfolgerung}

Die Messung hat den qualitativen Verlauf für den untersuchten Prozess der Aufnahme von Wasser in SPEEKSchichten und die sich dadurch ändernde Leitfähigkeit erfasst. Auch quantitativ sind die Werte mit Literaturwerten vergleichbar. Die Messung hat es insbesondere ermöglicht, die beiden zusammenhängenden Größen der Massenaufnahme von Wasser und der Leitfähigkeit parallel zu beobachten und somit direkt in Beziehung zu setzen.

Es ist dabei über den gesamten Verlauf der relativen Feuchtigkeit ein exponentieller Verlauf der Leitfähigkeit erkennbar. Folglich ist das Konzept der Messung der relativen Feuchtigkeit über die Leitfähigkeit von SPEEK gegeben.

Bevor jedoch ein solcher Sensor praktisch getestet werden kann, bedürfen einige gefundene Auffälligkeiten im Prozess weiterer Untersuchung. Es ist vor allem bezüglich der Langlebigkeit eines potentiellen Sensors zu klären, wie genau und wodurch nach der Trocknung der Proben eine geringere Wasseraufnahme und Leitfähigkeit als bei gleichen relativen Feuchtigkeit vor der Trocknung zu verzeichnen ist.

Um weitere Messungen mit der hier gezeigten Methodik für SPEEK durchführen zu können, müsste zudem die Ursache für den Abfall im $\Delta m$ - $r H$-Diagramm bei zirka $51,5 \%$ festgestellt werden.

\section{Literatur}

[1] WU, X. ; WANG, X. ; HE, G. ; BENZINGER, J.: Differences in Water Sorption and Proton Conductivity Between Nafion and SPEEK. In: Journal of Polymer Science: Polymer Physics 49 (2011), Nr. 20, S. 14371445

[2] SAUERBREY, G.: Verwendung von Schwingquarzen zur Wägung dünner Schichten und zur Mikrowägung. Zeitschrift der Physik 155 (1959), Nr. 2, S. 206-222

[3] FRITZE, H.: High-temperature bulk acoustic wave sensors. Measurement Science and Technology 22 (2010), Nr. 1, 012002 (28 Seiten)

[4] WULFMEIER, H.: Hochtemperatur-Dünnschichtkalorimetrie auf Basis piezoelektrischer Resonatoren, Technische Universität Clausthal, Dissertation 2017

[5] MUTHU LAKSHMI, R. T. S. ; CHOUDHARY, V. ; VARMA, I. K.: Sulphonated poly(ether ether ketone): Synthesis and characterisation. Journal of Material Science 40 (2005), Nr. 3, S. 629-636

[6] SHEN, J. ; GUO, Q. ; LI, D. ; TONG, J. ; LI, X.: Properties improvement of SPEEK based proton exchange membranes by doping of ionic liquids and Y2O3. Progress in Natural Science: Materials International 22 (2012), Nr. 3, S. 26-30 